Para enlazar con este artículo / To link to this article:

http://dx.doi.org/10.14198/fem.2019.34.13

Para citar este artículo / To cite this article:

Saldaña, María Nieves. «Del programa sectorial de estudios de las mujeres y del género a la integración de la perspectiva de género en los planes estatales de investigación científica y técnica y de innovación: avances, déficits y retos». En Feminismo/s, 34 (diciembre 2019): 289-315. DOI: 10.14198/fem.2019.34.13

\title{
DEL PROGRAMA SECTORIAL DE ESTUDIOS DE LAS MUJERES Y DEL GÉNERO A LA INTEGRACIÓN DE LA PERSPECTIVA DE GÉNERO EN LOS PLANES ESTATALES DE INVESTIGACIÓN CIENTÍFICA Y TÉCNICA Y DE INNOVACIÓN: AVANCES, DÉFICITS Y RETOS
}

\author{
FROM THE SECTORAL PROGRAM OF STUDIES OF WOMEN \\ AND GENDER TO THE INTEGRATION OF THE GENDER \\ PERSPECTIVE IN THE STATE PLANS OF SCIENTIFIC AND \\ TECHNICAL RESEARCH AND INNOVATION: PROGRESS, \\ DEFICITS AND CHALLENGES
}

\author{
MARÍA NiEVES SALDAÑA \\ Universidad de Huelva, Huelva \\ manieves@dpub.uhu.es \\ https://orcid.org/0000-0001-5869-6010
}

\section{Resumen}

Desde la aprobación de la Constitución en 1978, se han adoptado diversas medidas y acciones para la consecución de la igualdad de mujeres y hombres en el marco español de investigación, incorporándose la igualdad de género y la perspectiva de género en la Ley de la Ciencia, la Tecnología y la Innovación (2011), en la Estrategia Española de Ciencia y Tecnología y de Innovación (2013-2020), así como en los Planes Estatales de I+D+i. Sin embargo, actualmente se mantienen unos evidentes desequilibrios y sesgos de género que discriminan a las mujeres, así como un notable déficit de la integración del análisis de sexo/género en la investigación. Para su superación, es

Los contenidos de la revista se publican bajo una licencia de Creative Commons Reconocimiento 4.0 Internacional (CC BY 4.0)

Feminismo/s 34, diciembre 2019, pp. 289-315 
Del programa sectorial de estudios de las mujeres y del género a la integración de la perspectiva de género en los planes estatales de investigación científica y técnica y de innovación: avances, déficits y retos

necesaria la regulación en una disposición normativa de forma sistemática e integral de todos los principios, objetivos, acciones, medidas y sistema de financiación, así como de los mecanismos de seguimiento, evaluación y rendición de cuentas que han de implementarse para alcanzar la igualdad real y efectiva de mujeres y hombres en el marco español de investigación de conformidad con el Espacio Europeo de Investigación y el próximo Programa Marco de Investigación e Innovación «Horizonte Europa» (2021-2027).

Palabras clave: Investigación; España; Igualdad de género.

\begin{abstract}
Since the adoption of the Constitution in 1978, various measures and actions have been taken to achieve equality between women and men in the Spanish research framework, incorporating gender equality and the gender perspective in the Law of Science, Technology and Innovation (2011), in the Spanish Strategy for Science and Technology and Innovation (2013-2020), as well as in the National R\&D\&I Plans. However, there are currently evident gender imbalances and biases that discriminate against women, as well as a notable deficit in the integration of sex/gender analysis in research. To overcome it, it is necessary to regulate systematically and comprehensively all the principles, objectives, actions, measures and financing system, as well as the monitoring, evaluation and accountability mechanisms that must be implemented to achieve effectively the real and effective equality of women and men in the Spanish research framework in accordance with the European Research Area and the next Framework Program for Research and Innovation «Horizon Europe» (2021-2027).
\end{abstract}

Keywords: Research; Spain; Gender equality.

\title{
1. LA EMERGENCIA DEL «PROGRAMA SECTORIAL DE ESTUDIOS DE LAS MUJERES Y DEL GÉNERO»: 1978-1999
}

Tras la aprobación de la Constitución a finales de 1978, cuyo texto establece que los poderes públicos promoverán la ciencia y la investigación científica y técnica en beneficio del interés general (art. 44.2 CE), atribuyéndose al Estado la competencia exclusiva en materia de fomento y coordinación general de la investigación científica y técnica (art. 149.1.15. ${ }^{a}$ CE), la Ley 13/1986, de 14 de abril, de Fomento y Coordinación General de la Investigación Científica y Técnica, también conocida como «Ley de la Ciencia», supone un punto de inflexión importante, pues a partir de ella nace la organización de la política

Feminismo/s 34, diciembre 2019, pp. 289-315 
Del programa sectorial de estudios de las mujeres y del género a la integración de la perspectiva de género en los planes estatales de investigación científica y técnica y de innovación: avances, déficits y retos

de fomento de la I+D en España y su incardinación en la europea, poniéndose en marcha los Planes Naciones de Investigación Científica y Desarrollo Tecnológico, aunque habría que esperar una década para que se incluyesen específicamente acciones tendentes a la igualdad de mujeres y hombres. En efecto, ni en el I Plan Nacional de Investigación Científica y Desarrollo Tecnológico (PNID) (1988-1991), ni en el II Plan Nacional de I+D (19921995) se contiene acción alguna relativa a la promoción de la igualdad de mujeres y hombres, habría que esperar a la aprobación del III Plan para que la igualdad de género hiciera acto de presencia en la política científica nacional. Así, en el marco del III Plan Nacional de I+D (1996-1999) emerge el Programa Sectorial de Estudios de las Mujeres y del Género, cuyos objetivos son el fomento, promoción y apoyo de los estudios de las mujeres, la introducción de tales estudios en los currículos universitarios así como la formación de personas expertas en estas materias. Su articulación se implementa mediante un Convenio de Colaboración entre la Comisión Interministerial de Ciencia y Tecnología (CICYT) y el Instituto de la Mujer, adscrito al Ministerio de Trabajo y Asuntos Sociales. La duración del Programa es de cuatro años (19961999), está gestionado por el Instituto de la Mujer y financiado al 50\% por la CICYT y por el propio Instituto. Para su ejecución se publica anualmente una convocatoria de concesión de subvenciones competitivas destinadas a la realización de proyectos de investigación cuyos temas prioritarios deben enmarcarse en las líneas de investigación que incorporan una perspectiva de género en su tarea investigadora, incluyéndose las líneas prioritarias relativas a trabajo, educación, salud, violencia, medios de comunicación, medio ambiente y pobreza (CICYT 1996).

Sin duda, la inclusión del Programa Sectorial de Estudios de las Mujeres y del Género en el III Plan Nacional de I+D representa un avance importante para potenciar y promover la investigación en materia de igualdad de mujeres y hombres, sin embargo, tanto las cuantías destinadas al mismo como el número de los proyectos financiados fueron limitados y escasos, como reflejan las Memorias de Actividades del Plan correspondientes a los años 1996 a 1999. En efecto, a la primera convocatoria de 1996 se presentaron 86 proyectos, de los que se aprobaron solo 33, con un presupuesto global de 200 Mptas. (Millones de pesetas), algo más del 38\% de lo solicitado. Por lo que

Feminismo/s 34, diciembre 2019, pp. 289-315 
Del programa sectorial de estudios de las mujeres y del género a la integración de la perspectiva de género en los planes estatales de investigación científica y técnica y de innovación: avances, déficits y retos

se refiere a las áreas prioritarias, se concedieron 13 proyectos con una financiación global de 74,5 Mptas. en el área prioritaria dedicada al estudio de los procesos de redefinición de las pautas y objetos del conocimiento científico desde una óptica de género; 9 proyectos por valor de 62,6 Mptas. en el área de empleo y nuevas formas de trabajo; 8 por una cuantía de 45,5 Mptas. en el área de nuevas tecnologías aplicadas a la vida cotidiana, la salud, el bienestar, el medio ambiente y la comunicación; y 3 proyectos con una financiación total de 18,5 Mptas. en el área relativa al análisis de la eficacia y adecuación de las políticas públicas a los intereses y necesidades de las mujeres (CICYT 1998,111-112).

En cuanto al año 1997, se presentaron 59 proyectos de los que se aprobaron 22 (37\%), por un presupuesto global de 93 Mptas. Por lo que se refiere a las áreas prioritarias, en el área de trabajo se concedieron 10 proyectos (39,8 Mptas.), en la de educación se aprobaron 5 (15,8 Mptas.), en la de violencia 2 (15,8 Mptas.), en la de salud 3 (12,8 Mptas.) y en el área de medios de comunicación se concedieron 2 proyectos (8,5 Mptas.) (CICYT 1999,117-118).

En 1998 se aprobaron 29 de los 64 proyectos presentados (45,3\%) y las subvenciones concedidas alcanzaron la cifra de 110,5 Mptas. En relación con las áreas prioritarias, se aprobaron 9 proyectos en el área de trabajo, 6 en la de salud, 5 en las áreas de educación y pobreza, 3 en la de violencia y 1 en el área de medios de comunicación (CICYT 2001, 125-126).

Y en 1999 se aprobaron 35 de los 87 proyectos presentados (40,2\%), estando las subvenciones concedidas muy cerca de alcanzar los 130,6 Mptas., aprobándose 10 proyectos en el área de salud, 7 en el área de trabajo, 6 en la de educación, 5 en el área de pobreza, 3 en la de medio ambiente y 2 proyectos en las áreas de violencia y medios de comunicación (CICYT 2001, 139-140).

Como puede apreciarse en la Tabla 1, la tasa de éxito de los proyectos concedidos a lo largo de los cuatro años no alcanzó el 50\% ningún año, produciéndose una reducción drástica, de más del 50\%, en el importe total concedido en la convocatoria de 1997 respecto de la de 1996, manteniéndose, aunque en menor medida, tal decremento en 1998 (-45\%) y en 1999 $(-35 \%)$.

Feminismo/s 34, diciembre 2019, pp. 289-315 
Del programa sectorial de estudios de las mujeres y del género a la integración de la perspectiva de género en los planes estatales de investigación científica y técnica y de innovación: avances, déficits y retos

Tabla 1. Programa Sectorial de Estudios de las Mujeres y del Género.

Proyectos presentados y aprobados: 1996-1999

\begin{tabular}{|c|c|c|c|c|}
\hline \multirow{2}{*}{ Años } & Solicitados & \multicolumn{3}{|c|}{ Concedidos } \\
\cline { 2 - 5 } & Núm. & Núm. & $\begin{array}{c}\text { \% total de } \\
\text { aprobados }\end{array}$ & Mptas. \\
\hline 1996 & 86 & 33 & 38,4 & 200 \\
\hline 1997 & 59 & 22 & 37,3 & 93 \\
\hline 1998 & 64 & 29 & 45,3 & 110,5 \\
\hline 1999 & 87 & 35 & 40,2 & 130,6 \\
\hline
\end{tabular}

Elaboración propia según los datos publicados en las Memorias de Actividades de I+D (CICYT 1998, 1999, 2001)

\section{LAS LIMITACIONES DE LA «ACCIÓN ESTRATÉGICA DE FOMENTO DE LA IGUALDAD DE OPORTUNIDADES ENTRE MUJERES Y HOMBRES»: 2000-2010}

El Programa Sectorial de Estudios de las Mujeres y del Género pasa a constituirse en Acción Estratégica de Fomento de la Igualdad de Oportunidades entre Mujeres y Hombres en el IV Plan Nacional de I+D+i 2000-2003, vinculada a través del Área científico-tecnológica de Socioeconomía, que pasa a ser gestionada únicamente por el Ministerio de Trabajo y Asuntos Sociales a través del Instituto de la Mujer (CICYT 2000). No obstante, siguiendo la tónica del Plan de I+D 1996-1999, tanto las cuantías destinadas al mismo como los proyectos financiados fueron limitados y escasos. Así, a la convocatoria del año 2000 se presentaron 100 proyectos, por un total de 945,3 Mptas. de los que se aprobaron solamente 36 , por un total de 168,2 Mptas. (17,8\% del solicitado) (CICYT 2001, 150-151). Y a la convocatoria de 2001 se presentaron 128 solicitudes de financiación para proyectos de investigación (1.057,6 Mptas.), de las que se aprobaron 41 (155,2 Mptas.), situándose las tasas de éxito en el 32\% y 15\% respectivamente. Entre las áreas temáticas cuyos proyectos de investigación solicitados fueron financiados destacan educación (12 proyectos), salud (8), economía y empleo (7), violencia (5) y mujeres en situación de exclusión social (4). Aunque, los resultados globales, en comparación con los obtenidos en la convocatoria de 2000, experimentaron

Feminismo/s 34, diciembre 2019, pp. 289-315 
Del programa sectorial de estudios de las mujeres y del género a la integración de la perspectiva de género en los planes estatales de investigación científica y técnica y de innovación: avances, déficits y retos

un notable ascenso tanto en el número de proyectos (102 solicitados y 36 concedidos en 2000) como en el importe de los solicitados (935,6 Mptas.), sin embargo, la financiación aprobada sufrió un descenso del 7,7\% (168,2 Mptas.en 2000) y las tasas de éxito un retroceso de 3 puntos porcentuales en ambos casos (CICYT 2003, 199-200).

En el año 2002 se solicitaron un total de 132 proyectos, por una cuantía de 6,2 M€, siendo concedidos 50 proyectos por una cuantía total de 1,0 M€, estando adscritos principalmente a las áreas de educación (22\%), economía y empleo (20\%), violencia (14\%), salud y mujeres en situación de exclusión social (10\%) (CICYT 2004, 184-185). Y en 2003 se solicitaron un total de 146 proyectos, por una cuantía de 6,8 M€, de los que se concedieron 37 proyectos, un $26 \%$ menos que el año anterior, con una financiación aprobada de 1,0 M€, lo que supone el 1,5\% menos que en 2002 (CICYT 2005, 213-214).

Como puede apreciarse en la Tabla 2, a lo largo de los cuatro años de vigencia de la Acción Estratégica, la tasa de éxito de los proyectos concedidos no alcanza el 40\% ningún año, estando por debajo del $20 \%$ los importes asignados respecto del total solicitado.

Tabla 2. Acción Estratégica de Fomento de la Igualdad de Oportunidades entre Mujeres y Hombres. Proyectos presentados y probados: 2000-2003

\begin{tabular}{|c|c|c|c|c|c|c|}
\hline \multirow{2}{*}{ Años } & \multicolumn{2}{|c|}{ Solicitados } & \multicolumn{4}{c|}{ Concedidos } \\
\cline { 2 - 7 } & Núm. & $\begin{array}{c}\text { Mptas./ } \\
\text { M€ }\end{array}$ & Núm. & $\begin{array}{c}\text { \% total de } \\
\text { aprobados }\end{array}$ & $\begin{array}{c}\text { Mptas. / } \\
\text { M€ }\end{array}$ & $\begin{array}{c}\text { \% total } \\
\text { concedido }\end{array}$ \\
\hline 2000 & 100 & 945,3 & 36 & 36 & 168,2 & 17,7 \\
\hline 2001 & 128 & $1.057,6$ & 41 & 32 & 155,2 & 14,6 \\
\hline 2002 & 132 & 6,2 & 50 & 37,8 & 1,0 & 16,3 \\
\hline 2003 & 146 & 6,8 & 38 & 25,3 & 1,0 & 14,7 \\
\hline
\end{tabular}

Elaboración propia según los datos publicados en las Memorias de Actividades de I+D+i (CICYT, 2001, 2003, 2004, 2005)

Posteriormente, en el V Plan Nacional de Investigación Científica, Desarrollo e Innovación Tecnológica 2004-2007 se propone un crecimiento del gasto en investigación sostenido y asequible, pretendiendo alcanzarse un gasto en I+D del 1,22\% del PIB en 2005 y un 1,4\% en 2007, para cuya

Feminismo/s 34, diciembre 2019, pp. 289-315 
Del programa sectorial de estudios de las mujeres y del género a la integración de la perspectiva de género en los planes estatales de investigación científica y técnica y de innovación: avances, déficits y retos

consecución se estima que la Administración debería destinar a I+D+i en los dos primeros años un $20 \%$ más con respecto a 2003, pasando de dedicar 4.000 M€ anuales a más de 4.800 M€ (CICYT 2003). Sin embargo, se reducen las cuantías y el número en relación con los proyectos presentados a la convocatoria del Instituto de la Mujer en el marco de la Acción Estratégica para el fomento de la igualdad de oportunidades entre mujeres y hombres. Así, a la convocatoria de 2004 se presentaron 180 proyectos, de los que se aprobó solo el 13\%, con una financiación total por valor de 897,0 k€ (miles de euros), representando el 9\% del importe total presentado respecto al año 2003, pues en 2004 se presentó casi una cuarta parte más de proyectos (23,3\%), y el $47,5 \%$ más de subvenciones, pero en cambio se aprobaron menos proyectos y subvenciones $(-35,1 \%$ y $-11,6 \%)$. Los proyectos financiados se enmarcan en las líneas temáticas centradas en la promoción de la calidad de vida de las mujeres (29\% del total de proyectos), igualdad entre hombres y mujeres en la vida económica y fomento de la igualdad civil (16\%) y políticas públicas y transmisión de valores y actitudes igualitarias (12\%) (CICYT 2006, 203). Asimismo, en la convocatoria de 2005 se solicitó un total de 153 proyectos, por una cuantía de 7,2 M€, siendo concedidos solamente 34 proyectos por una cuantía de 1,1 M€, estando centrados principalmente en las líneas temáticas relativas a políticas públicas, igualdad entre mujeres y hombres en la vida económica, participación en la toma de decisiones, conciliación de la vida personal y social de las mujeres, violencia de género y colectivos de mujeres en riesgo de exclusión social (CICYT 2007, 253-254).

La escasa relevancia que se le otorga a la Acción Estratégica de Fomento de la Igualdad de Oportunidades entre Mujeres y Hombres, se refleja también en la convocatoria de 2006, pues esta acción estratégica representa solo el $0,4 \%$ del total de programas nacionales y acciones estratégicas aprobadas y el 0,1\% de la financiación asignada al Plan. Así, en 2006 se presentaron 146 proyectos, de los que se aprobaron $36(24,7 \%)$, siendo la financiación solicitada por importe total de 7.575,7 M€ en subvenciones, aunque la financiación concedida fue solo de $1.098 \mathrm{M} €$, lo que supone una tasa de éxito del 14,5\%, inferior incluso al 15,3\% de éxito obtenido en 2005, estando los proyectos enmarcados principalmente en las áreas temáticas relativas a políticas públicas, igualdad entre mujeres y hombres en la vida económica, participación

Feminismo/s 34, diciembre 2019, pp. 289-315 
Del programa sectorial de estudios de las mujeres y del género a la integración de la perspectiva de género en los planes estatales de investigación científica y técnica y de innovación: avances, déficits y retos

en la toma de decisiones, transmisión de actitudes y valores igualitarios y medios de comunicación (CICYT 2008, 303-305).

Y en 2007, último año del Plan Nacional de I+D+i 2004-2007, se produce incluso un decremento del 21,3\% en el presupuesto total asignado a la Acción Estratégica de Fomento de Igualdad de Oportunidades entre Mujeres y Hombres. Así, en 2007 se presentaron 163 proyectos de I+D, de los que se aprobaron 42 (25,8\%), alcanzando la financiación solicitada el importe total de 9.297,3 M€ en subvenciones, aunque la financiación concedida fue solo de $864 \mathrm{k} €$, lo que supone una tasa de éxito del 9,3\% de la cantidad solicitada, inferior en más de 5 puntos porcentuales a la de 2006 (CICYT 2009, 353-355).

Como puede apreciarse en la Tabla 3, a lo largo de los cuatro años de vigencia de la Acción Estratégica, la tasa de éxito de los proyectos concedidos no alcanza el 26\% ningún año, estando por debajo del $16 \%$ los importes asignados respecto del total solicitado.

Tabla 3. Acción Estratégica de Fomento de la Igualdad de Oportunidades entre Mujeres y Hombres. Proyectos presentados y aprobados: 2004-2007

\begin{tabular}{|c|c|c|c|c|c|c|}
\hline \multirow{2}{*}{ Años } & \multicolumn{2}{|c|}{ Solicitados } & \multicolumn{4}{c|}{ Concedidos } \\
\cline { 2 - 7 } & Núm. & M€ & Núm. & $\begin{array}{c}\text { \% total de } \\
\text { aprobados }\end{array}$ & K€/M€ & $\begin{array}{c}\% \text { total } \\
\text { concedido }\end{array}$ \\
\hline 2004 & 180 & 10 & 23 & 13 & 897,6 & 9 \\
\hline 2005 & 153 & 7,2 & 34 & 22,2 & 1,1 & 15,3 \\
\hline 2006 & 146 & 7,5 & 36 & 24,7 & 1,09 & 14,5 \\
\hline 2007 & 163 & 9,3 & 42 & 25,8 & 864 & 9,3 \\
\hline
\end{tabular}

Elaboración propia según los datos publicados en las Memorias de Actividades de I+D+i (CICYT, 2004, 2005, 2006, 2007)

Por esto no extraña que en el Informe SISE 2006 se afirme que la Acción Estratégica de Fomento de Igualdad de Oportunidades entre Mujeres y Hombres presenta una escasa financiación en cuanto a los fondos y el número de proyectos que se subvencionan, así como a la imposibilidad de obtener fondos por vías indirectas, especialmente a través de las convocatorias complementarias, lo que se traduce en la dificultad de difusión de los resultados, el escaso aumento del conocimiento en la materia así como las dificultades

Feminismo/s 34, diciembre 2019, pp. 289-315 
Del programa sectorial de estudios de las mujeres y del género a la integración de la perspectiva de género en los planes estatales de investigación científica y técnica y de innovación: avances, déficits y retos

en la formación de investigadores e investigadoras en el área de conocimiento sobre Estudios de las Mujeres, Feministas y del Género. Para su superación, se recomienda aumentar la dotación de la Acción Estratégica transversal de fomento de la igualdad de oportunidades entre mujeres y hombres mediante la participación de la Dirección General de Investigación (DGI), manteniéndose la del Instituto de la Mujer; priorizar el desarrollo de actuaciones que faciliten la incorporación y mantenimiento de la mujer en la carrera científica, de forma que se facilite la incorporación y mantenimiento de un mayor porcentaje de mujeres al campo de la ciencia y la tecnología; y contar con la participación y asesoramiento de mujeres en todas las fases del desarrollo de las políticas y, en particular, en las diversas comisiones que articulan el sistema científico español a nivel académico, ministerial, etc., debiendo fomentarse la utilización de un lenguaje no sexista en todos los documentos del Plan Nacional (FECYT 2007, 222, 228).

Un año más tarde se aprueba el VI Plan Nacional de Investigación Científica, Desarrollo e Innovación Tecnológica para el período 2008-2011, contemplándose la incorporación de la igualdad de género con carácter transversal, pues, en la medida en que uno de los principios básicos de la Estrategia Nacional de Ciencia y Tecnología (ENCYT) de 2007 hace referencia a la plena e igual incorporación de la mujer (CICYT 2007, 4), las actuaciones financiadas públicamente en $\mathrm{I}+\mathrm{D}+\mathrm{i}$ han de contemplar el principio de igualdad y la perspectiva de género, de conformidad, además, con lo previsto en la entonces recientemente aprobada Ley Orgánica 3/2007, de 22 de marzo, para la Igualdad Efectiva de Mujeres y Hombres (LOI), cuyo art. 25 establece que en el ejercicio de sus respectivas competencias, las Administraciones públicas fomentarán la investigación sobre el significado y alcance de la igualdad entre mujeres y hombres, promoviéndose la realización de estudios e investigaciones especializadas en la materia. Así, debido a su carácter transversal, el principio de igualdad ha de impregnar toda la filosofía y los programas de ayudas del Plan Nacional de I+D+i 2008-2011, promoviéndose la realización de estudios e investigaciones especializadas sobre el significado y alcance de la igualdad entre mujeres y hombres, la elaboración de los informes de resultados e impacto de las actuaciones financiadas al amparo del Plan desde la perspectiva de género (incluyendo sistemáticamente la variable de sexo en las estadísticas, encuestas y recogida de datos que se realicen), así como

Feminismo/s 34, diciembre 2019, pp. 289-315 
Del programa sectorial de estudios de las mujeres y del género a la integración de la perspectiva de género en los planes estatales de investigación científica y técnica y de innovación: avances, déficits y retos

la presencia equilibrada de mujeres y hombres en los órganos de selección y comisiones de valoración (CICYT 2007, 58).

Pese a todo, no se menciona como tal en el Plan Nacional de I+D+i 20082011 la Acción Estratégica de Fomento de la Igualdad de Oportunidades entre Mujeres y Hombres, desapareciendo las correspondientes referencias en las Memorias anuales. Por esto no extraña el análisis crítico que se realiza en el Informe SISE 2008, constatándose la tendencia decreciente en cuanto a su financiación, poniéndose de manifiesto la desproporción que hay entre la necesidad de extender la perspectiva igualitaria y de género a todo el conjunto del sistema de I+D+i y la realidad concreta de la Acción Estratégica que tiene por objetivo prioritario la igualdad de hombres y mujeres. Así, aunque desde 1997 se ha producido un aumento continuo en la asignación presupuestaria para la financiación pública de $\mathrm{I}+\mathrm{D}$, pues la dotación inicial del Programa de Gasto 46 de los Presupuestos Generales del Estado (Investigación científica, Desarrollo e Innovación) se ha incrementado durante los cuatro años de vigencia un $103 \%$, lo que ha supuesto alcanzar en 2007 un total de 8.124 $\mathrm{M} €$, sin embargo, en relación con la subvención destinada a los Estudios de Género, se ha producido una disminución de más del 20\% del total inicialmente asignado en 1996, representando los fondos asignados en el año 2007 únicamente el 0,0098\% respecto de total asignado al Plan Nacional. Estos datos son manifiestamente contrarios a las previsiones establecidas en LOI, por esto se recomienda que se articulen los medios que permitan que la Acción Estratégica de Fomento de la Igualdad de Oportunidades entre Mujeres y Hombres recupere su carácter de $\mathrm{I}+\mathrm{D}+\mathrm{i}$ formal que nunca debió perder, adaptándose el conjunto del sistema de $\mathrm{I}+\mathrm{D}+\mathrm{i}$ a las previsiones normativas de la LOI, tanto en cuanto a contenidos, como en cuanto a la adopción de la perspectiva de género en otras líneas de investigación, insistiéndose en la visibilización estadística de las mujeres en el sistema I+D+i y en su participación equilibrada en los distintos ámbitos de actuación (Observatorio Permanente del Sistema Español de Ciencia-Tecnología-Sociedad 2009, 202, 203, 210).

Sin embargo, en el Informe SISE 2009, se constata que no se realiza una convocatoria independiente en relación a las ayudas a la investigación, incorporándose como línea prioritaria en la convocatoria de las acciones complementarias a los proyectos de investigación fundamental no-orientada

Feminismo/s 34, diciembre 2019, pp. 289-315 
Del programa sectorial de estudios de las mujeres y del género a la integración de la perspectiva de género en los planes estatales de investigación científica y técnica y de innovación: avances, déficits y retos

del Programa Nacional de Proyectos de Investigación Fundamental (LIA de Proyectos de $\mathrm{I}+\mathrm{D}+\mathrm{i}$ ), indicándose únicamente en una tabla anexa que el importe total relativo a las ayudas para la realización de estudios feministas ascendió a $845.900 €$, algo menos incluso que en 2008 (Observatorio Español de I+D+i (ICONO) 2011, 28). Y en el Informe SISE 2010, el importe total relativo a las ayudas para la realización de Estudios Feministas en el Área de Humanidades y Ciencias Sociales ascendió únicamente a $446.000 €$, casi la mitad de lo concedido en 2009, representando el 16\% respecto de lo solicitado (Observatorio Español de I+D+i (ICONO) 2011, 88-89).

En total, según los datos publicados en Científicas en Cifras 2013, durante el período 2009-2012, la tasa de éxito de los proyectos concedidos en el marco del Programa de Estudios de las Mujeres, Feministas y del Género (Proyectos FEM) representa el 35,8\%, 19 puntos porcentuales menos que la tasa de éxito correspondiente a los proyectos concedidos en el total del Plan Nacional de I+D+i $(54,1 \%)$, concediéndose únicamente el 13,3\% de la financiación solicitada, por un importe de 1,7 M€, 23 puntos porcentuales menos respecto del total asignado a todos los proyectos del Plan nacional de I+D+i $(36,5 \%)$, que ascendió a 1.488,7 M€, como refleja el Gráfico 1.

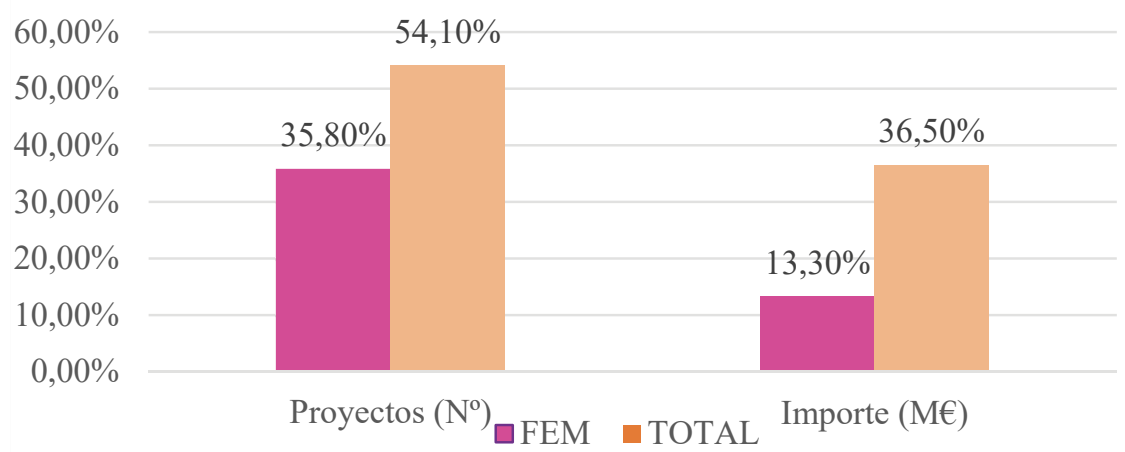

Gráfico 1. Tasa de éxito e importe concedido respecto a importe solicitado en proyectos presentados al Programa FEM en relación al total del Plan Nacional de I+D+i 2009-2012 (Unidad de Mujeres y Ciencia 2014, 78)

Feminismo/s 34, diciembre 2019, pp. 289-315 
Del programa sectorial de estudios de las mujeres y del género a la integración de la perspectiva de género en los planes estatales de investigación científica y técnica y de innovación: avances, déficits y retos

\section{LA INCORPORACIÓN DEL ENFOQUE DE GÉNERO CON CARÁCTER TRANSVERSAL EN EL SISTEMA ESPAÑOL DE CIENCIA, TECNOLOGÍA E INNOVACIÓN: 2011}

Uno de los aspectos más relevantes y novedosos de la Ley 14/2011, de 1 de junio, de la Ciencia, la Tecnología y la Innovación (LCTI) es la inclusión entre sus objetivos de la promoción de la inclusión de la perspectiva de género como categoría transversal en la ciencia, la tecnología y la innovación, así como una presencia equilibrada de mujeres y hombres en todos los ámbitos del Sistema Español de Ciencia, Tecnología e Innovación (SECTI) (art. 2.k LCTI), siendo en la Disposición adicional decimotercera de la LCTI donde se regula específicamente la implantación de la perspectiva de género. Así, en primer lugar se exige el cumplimiento del principio de presencia o composición equilibrada de género establecido en la LOI (art. 4.4), de manera que la composición de los órganos, consejos y comités regulados en la LCTI, así como de los órganos de evaluación y selección del SECTI, se ajuste a los principios de composición y presencia equilibrada entre mujeres y hombres (punto 1).

Asimismo, en la disposición adicional se contempla la integración de la perspectiva de género con carácter transversal en todo proceso investigador, estableciéndose que la Estrategia Española de Ciencia y Tecnología y el Plan Estatal de Investigación Científica y Técnica promoverán la incorporación de la perspectiva de género como una categoría transversal en la investigación y la tecnología, de manera que su relevancia sea considerada en todos los aspectos del proceso, incluidos la definición de las prioridades de la investigación científico-técnica, los problemas de investigación, los marcos teóricos y explicativos, los métodos, la recogida e interpretación de datos, las conclusiones, las aplicaciones y los desarrollos tecnológicos y las propuestas para estudios futuros, promoviéndose igualmente los estudios de género y de las mujeres, así como medidas concretas para estimular y dar reconocimiento a la presencia de mujeres en los equipos de investigación (Disposición adicional decimotercera LCTI, punto 2).

Igualmente, la LCTI integra la igualdad de género en el instrumento de captación de datos y análisis para la elaboración y seguimiento de la Estrategia Española de Ciencia y Tecnología, de la Estrategia Española de Innovación y de sus planes de desarrollo, estableciendo que el Sistema de Información

Feminismo/s 34, diciembre 2019, pp. 289-315 
Del programa sectorial de estudios de las mujeres y del género a la integración de la perspectiva de género en los planes estatales de investigación científica y técnica y de innovación: avances, déficits y retos

sobre Ciencia, Tecnología e Innovación recogerá, tratará y difundirá los datos desagregados por sexo e incluirá indicadores de presencia y productividad (Disposición adicional decimotercera LCTI, punto 3).

De igual manera, la LCTI articula criterios de actuación para la eliminación de posibles sesgos discriminatorios de género en los procesos de selección y evaluación, estableciendo expresamente que los procedimientos de selección y evaluación del personal investigador al servicio de las Universidades públicas y de los Organismos Públicos de Investigación (OPIs) de la Administración General del Estado, y los procedimientos de concesión de ayudas y subvenciones por parte de los agentes de financiación de la investigación, establecerán mecanismos para eliminar los sesgos de género que incluirán la introducción de procesos de evaluación confidencial para eliminar cualquier discriminación por razón de nacimiento, raza, sexo, religión o cualquier otra condición o circunstancia personal o social (Disposición adicional decimotercera LCTI, punto 4). En relación con este punto, el legislador es consciente de que diversos estudios han puesto de manifiesto la existencia de sesgos discriminatorios de género en los procesos de selección, evaluación y promoción que afectan especialmente a las mujeres, siendo necesario introducir diversos mecanismos que eliminen tales sesgos, concienciando a las entidades de la necesidad y los beneficios de basar su selección en los conocimientos y competencias de la persona, informando a las mujeres susceptibles de ser especialmente víctimas de los sesgos discriminatorios en los procesos de selección y habilitando mecanismos para garantizar procesos de selección neutros de género durante las distintas fases del mismo (League of European Research Universities (LERU) 2018; European Commission 2017).

\section{LA PERSISTENTE BAJA TASA DE ÉXITO DEL PROGRAMA DE ESTUDIOS FEMINISTAS, DE LA MUJER Y DE GÉNERO (PROGRAMA FEM): 2013-2016}

Conforme a los mandatos de la LCTI, la Estrategia Española de Ciencia y Tecnología y de Innovación (EECTI) 2013-2020 incluye entre sus principios básicos la incorporación de la perspectiva de género en las políticas públicas de I+D+i de las Administraciones Públicas para corregir la pérdida de capital humano asociada a la desigual incorporación de las mujeres y su desarrollo

Feminismo/s 34, diciembre 2019, pp. 289-315 
Del programa sectorial de estudios de las mujeres y del género a la integración de la perspectiva de género en los planes estatales de investigación científica y técnica y de innovación: avances, déficits y retos

profesional en los ámbitos de la investigación científica y técnica, tanto en el sector público como en el empresarial, conllevando este principio la incorporación de la perspectiva de género en los contenidos de la investigación científica, técnica y de la innovación para que se enriquezca el proceso creativo y la obtención de resultados (Ministerio de Economía y Competitividad 2013, 5).

Ese mismo año se aprueba el Plan Estatal de Investigación Científica y Técnica y de Innovación 2013-2016, que responde a los objetivos establecidos en la EECTI 2013-2020 y a sus ejes prioritarios, sin olvidar los retos que se plantean a nivel de la Unión Europea en el Programa Marco de Investigación e Innovación «Horizonte 2020» (Unión Europea 2013) en relación con el desarrollo y consolidación del Espacio Europeo de Investigación (EEI) (Ministerio de Economía y Competitividad 2013, 7-12). Sin embargo, en el Plan Estatal únicamente se hace mención de manera expresa al enfoque de género en el Programa Estatal de I+D+i Orientada a los Retos de la Sociedad, señalándose en el marco del Reto sobre Cambios e Innovaciones Sociales que la investigación que incorpore la perspectiva de género tendrá un carácter transversal y como tal se incorporará en el desarrollo de la investigación científico-técnica y de las innovaciones orientadas a la búsqueda de soluciones en todos los retos de la sociedad, incluyéndose solamente entre una de las prioridades científico-técnicas y sociales que se contemplan dentro de este Reto, relativa al crecimiento económico y la generación de empleo, las tasas de ocupación femenina y las mujeres y el desarrollo científico, tecnológico e innovación (Ministerio de Economía y Competitividad 2013, 27-28 y 37).

Por esto no sorprende que en el Plan de Actuación Anual de 2013 del Plan Estatal de I+D+i 2013-2016 no haya ninguna mención relacionada con la inclusión de la perspectiva de género (Ministerio de Economía y Competitividad 2013). Y que en los posteriores Programas de Actuación Anual del Plan Estatal correspondientes a 2014, 2015, 2016 y 2017 tampoco haya ninguna mención a la inclusión de la perspectiva de género (Ministerio de Economía y Competitividad 2014, 2015, 2016, 2017).

No obstante, según los datos publicados en Científicas en Cifras 2015, durante el período 2013-2014 se constata un retroceso en las propuestas presentadas al Programa FEM, dado que ha pasado a ser más relevante la menor tasa de éxito (20\%) y el menor importe proporcional de financiación concedida respecto al total solicitado (6\%) en este programa comparado con el

Feminismo/s 34, diciembre 2019, pp. 289-315 
Del programa sectorial de estudios de las mujeres y del género a la integración de la perspectiva de género en los planes estatales de investigación científica y técnica y de innovación: avances, déficits y retos

conjunto de las propuestas presentadas a las demás convocatorias 2013-2014, cuya tasa de éxito es del $40 \%$ y el importe proporcional concedido del $36 \%$ (Gráfico 2), aumentándose, en consecuencia, la brecha ya existente respeto de las convocatorias 2009-2012, siendo las áreas con mayor tasa de éxito las de Psicología, Historia y Arte, Biomedicina, Ciencias de la Educación, Filología y Filosofía. Y en cuanto a la pregunta introducida por primera vez en el formulario de solicitud de ayudas de proyectos en los programas de Excelencia y Retos a fin de promover la integración del análisis de sexo y/o género en el contenido de la investigación (¿Cree que el sexo (hombre/mujer) de los temas analizados puede afectar a los resultados de la investigación?) en las convocatorias de 2013 y 2014, solo el $9 \%$ de las propuestas presentadas y el 6-7\% de las concedidas respondieron afirmativamente a la pregunta, siendo las áreas científico-tecnológicas donde más se presentaron y concedieron estas propuestas, así como las de Ciencias Sociales, Ciencias Médicas y de la Salud, y donde menos las de Ciencias Naturales y Ciencias agrícolas (Unidad de Mujeres y Ciencia 2016, 101).

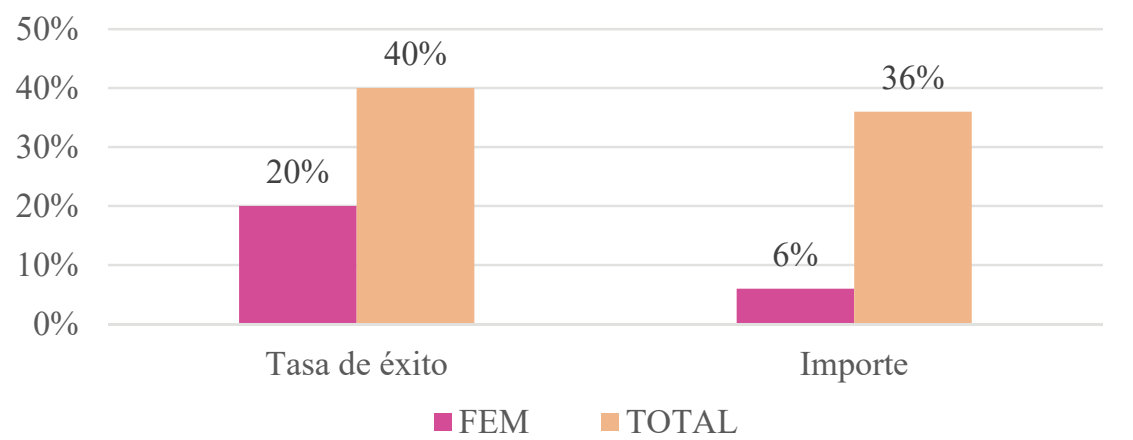

Gráfico 2. Comparación de tasa de éxito e importe concedido respecto a importe solicitado en propuestas presentadas al Programa FEM y total de propuestas presentadas a las convocatorias de proyectos I+D+i 2013-2014 (Unidad de Mujeres y Ciencia 2016, 100)

Igualmente, atendiendo a los datos recientemente publicados en Científicas en Cifras 2017, aunque se produce una cierta mejora de las tasas de éxito en los proyectos específicos del Programa FEM, pues las cifras agregadas de las convocatorias de los años 2015-2016 muestran que ha mejorado en 9 puntos

Feminismo/s 34, diciembre 2019, pp. 289-315 
Del programa sectorial de estudios de las mujeres y del género a la integración de la perspectiva de género en los planes estatales de investigación científica y técnica y de innovación: avances, déficits y retos

la tasa de éxito (29\%), y en 5 puntos el importe proporcional de financiación concedida respecto a la solicitada ( $11 \%)$, sin embargo, sigue existiendo una brecha relevante en este programa comparado con el conjunto de las propuestas presentadas a las demás convocatorias 2015-2016, cuya tasa de éxito es del 45\% y el importe proporcional concedido del 36\% (Gráfico 3). Asimismo, solo el 13\% de las propuestas presentadas y el $9 \%$ de las concedidas han respondido afirmativamente a la pregunta sobre relevancia del análisis de sexo/género para el contenido del proyecto, lo que supone una leve mejora de 4 y 2 puntos porcentuales, respectivamente, desde el periodo 2013-2014, manteniéndose las áreas científico-tecnológicas donde más se presentaron y concedieron estas propuestas así como en las de Ciencias Sociales y Ciencias Médicas y de la Salud (Unidad de Mujeres y Ciencia 2018, 124-127).

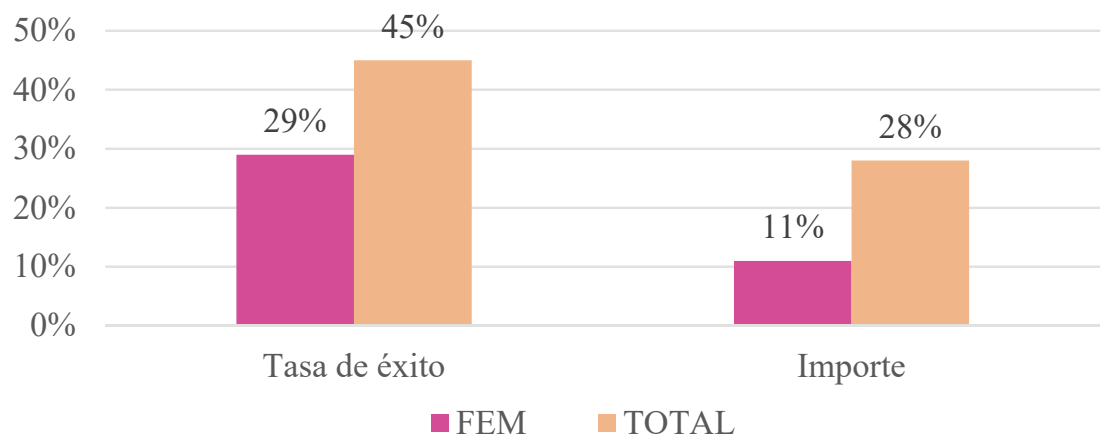

Gráfico 3. Comparación de tasa de éxito e importe concedido respecto a importe solicitado en propuestas presentadas al Programa FEM y total de propuestas presentadas a las convocatorias de proyectos I+D+i 2015-2016 (Unidad de Mujeres y Ciencia 2018, 126)

\section{EL RETO DE IMPULSAR LA IGUALDAD DE GÉNERO Y LA INTEGRACIÓN DEL ANÁLISIS DE GÉNERO EN LA INVESTIGACIÓN (IAGI): 2016-2020}

Al finalizar el período de vigencia del Plan Estatal de Investigación Científica y Técnica y de Innovación 2013-2016 se ponen de manifiesto las dificultades y retos que tiene que afrontar el SECTI, especialmente atendiendo a su

Feminismo/s 34, diciembre 2019, pp. 289-315 
Del programa sectorial de estudios de las mujeres y del género a la integración de la perspectiva de género en los planes estatales de investigación científica y técnica y de innovación: avances, déficits y retos

convergencia en el marco del EEI (Ministerio de Hacienda y Administraciones Públicas 2014; European Commission 2017, 2014). En este sentido, hay que tener en cuenta que el Consejo Europeo, en sus conclusiones sobre el EEI de 2014, incluye la elaboración de una Hoja de Ruta Europea que, junto a las Hojas de Ruta nacionales, identifiquen una serie de medidas necesarias para impulsar dicho Espacio (Council of the European Union 2014). Por lo que aquí interesa y en la medida en que desde la publicación del conocido «Informe ETAN» (European Commission 2000), la política de la Unión Europea en el ámbito de la investigación y la innovación incluye entre sus prioridades la igualdad de mujeres y hombres y la incorporación de la perspectiva de género en la investigación (Council of the European Union 2015), como se refleja en el vigente Programa Marco de Investigación e Innovación «Horizonte 2020» (Unión Europea 2013), la Prioridad 4 de la «Hoja de Ruta del EEI 2015-2020» está centrada en la «Igualdad de género e incorporación de la dimensión de género en la investigación», dirigiéndose expresamente a la legislación nacional para que se adopten medidas efectivas que aborden los desequilibrios de género en las instituciones de investigación y los órganos de toma de decisiones y se integre mejor la dimensión de género en las políticas, programas y proyectos de investigación (Council of the European Union 2015).

En consecuencia, la Prioridad 4 de la Hoja de Ruta del EEI en España 2016-2020 incluye el impulso de la igualdad de género y la integración de la perspectiva de género en la investigación (European Commission 2016, 4), agrupándose las medidas específicas en torno en dos amplios objetivos. El primero se centra en la revisión y adaptación de los procedimientos y criterios utilizados en las convocatorias públicas de I+D+i para evitar el sesgo de género, lograr un mayor equilibrio de género y mejorar la integración de la dimensión de género en las propuestas a ser financiadas, incluyéndose distintas medidas, especialmente: revisar e incorporar criterios sensibles al género en el campo de la evaluación científica del desempeño, convocatorias de becas de capacitación y ayudas de movilidad y reclutamiento; actualizar e incluir los nuevos criterios de la Integración del Análisis de Género en la Investigación (IAGI) (Unidad de Mujeres y Ciencia 2017) en las subvenciones de I+D+i para proyectos, según el Vademecum de Igualdad de Género de Horizonte 2020 (European Commission 2014) y las recomendaciones de

Feminismo/s 34, diciembre 2019, pp. 289-315 
Del programa sectorial de estudios de las mujeres y del género a la integración de la perspectiva de género en los planes estatales de investigación científica y técnica y de innovación: avances, déficits y retos

GENDER-NET (GENDER-NET 2016), que permitan identificar los temas en los que es esencial aplicar el análisis integrado de género; mejorar los criterios y requisitos para la composición de la comisión de evaluación de I+D+i con el objetivo de evitar el sesgo de género y promover la evaluación de la implementación del análisis integrado de género en la investigación; actualizar las bases de datos nacionales e internacionales que faciliten la ubicación de las y los investigadores, especialmente en los campos científicos que muestran una representación insuficiente, y la inclusión de personal de investigación experto en estudios de género y en estudios de impacto de la investigación de género; e incluir criterios de selección para ayudas de $\mathrm{I}+\mathrm{D}+\mathrm{i}$ que promuevan la igualdad de género en equipos, instituciones y en el contenido de las propuestas (European Commission 2016, 17).

Y en relación con el segundo objetivo, centrado en el desarrollo de directrices, promoción de mejores prácticas y capacitación para mejorar la implementación de políticas de igualdad de género, en sus dos aspectos, en centros públicos de investigación y agencias de financiación de I+D+i, y en cuanto a su monitorización y evaluación, se incluyen las siguientes actuaciones: diseñar e implementar un plan de capacitación dirigido a gestores de nivel medio y superior de centros públicos de investigación, sus órganos de gobierno, sus comités de control y evaluación; desarrollar estructuras de igualdad de género en los organismos públicos de investigación y en la Agencia Estatal de Investigación, mejorando la organización institucional a nivel nacional; fomentar un área conjunta de conocimiento y un sistema de acreditación para estudios de género y directrices, criterios, incentivos y mecanismos para desarrollar el sistema de producción de conocimiento relacionado con el género; desarrollar directrices y manuales que coadyuven a difundir buenas prácticas para el desarrollo de servicios y recursos orientados a conciliar el trabajo y la vida en los centros de investigación, con el objetivo de evitar sesgos y barreras relacionadas con el género en la movilidad del personal de investigación y la promoción de una mayor estabilidad y prestigio profesional; desarrollar directrices para la actualización de procedimientos y criterios en las convocatorias de propuestas, reglamentos y normas específicas de organizaciones públicas de investigación destinadas a garantizar la igualdad de género en la evaluación de candidaturas (selección, contratación y promoción), así como en la composición de los comités de evaluación; y mejorar la monitorización,

Feminismo/s 34, diciembre 2019, pp. 289-315 
Del programa sectorial de estudios de las mujeres y del género a la integración de la perspectiva de género en los planes estatales de investigación científica y técnica y de innovación: avances, déficits y retos

medición, indicadores y sistemas de rendición de cuentas de las actividades y los resultados en igualdad de género en los centros de investigación y organismos de financiación, incluyéndose en los informes de actividades anuales la inclusión de datos desagregados por sexo para todas las variables del análisis (European Commission 2016, 18).

Por esto no extraña que el nuevo Plan Estatal de Investigación Científica y Técnica y de Innovación 2017-2020, mencione en el Objetivo 5, relativo a promover un modelo de $\mathrm{I}+\mathrm{D}+\mathrm{i}$ abierto y responsable apoyado en la participación de la sociedad, la inclusión de la perspectiva de género en las actuaciones de $\mathrm{I}+\mathrm{D}+\mathrm{i}$ financiadas, así como la aplicación de criterios de paridad en las distintas comisiones de evaluación, comités y órganos de gestión y gobernanza del Plan Estatal y de las ayudas asociadas al mismo. Asimismo, se contempla el estímulo de las vocaciones científicas y tecnológicas, especialmente en el área de las ciencias y la ingeniería, y de la cultura científica e innovadora de la sociedad, prestando especial atención a medidas destinadas a corregir la brecha de género. Igualmente, entre las prioridades que se contemplan para la ejecución del Programa Estatal de Promoción del Talento y su Empleabilidad en I+D+i se incluye la adopción de medidas destinadas a corregir los desequilibrios de género en el acceso y promoción de las mujeres a lo largo de la carrera investigadora, contribuyendo todas las ayudas incluidas en el mismo a evitar la discriminación en el acceso a las ayudas por razones de género/sexo (Ministerio de Industria, Economía y Competitividad 2017, 32, 35, 40).

Por otra parte, entre los objetivos prioritarios del Programa Estatal de I+D+i Orientada a los Retos de la Sociedad, se incluye la integración de la dimensión de género, siempre que sea de aplicación, como una variable relevante de carácter transversal en la definición y desarrollo de los contenidos de la investigación. Así, en el marco de este Programa Nacional se contemplan unas «Ayudas para el fomento de la cultura científica y de la innovación», que están destinadas a la cofinanciación de actividades con la finalidad de impulsar la progresiva implantación de un modelo de ciencia en abierto, la participación ciudadana en las actividades de investigación científica y técnica, el estímulo de las vocaciones científicas y tecnológicas y el emprendimiento y la cultura científica y tecnológica de la sociedad, prestando especial atención a medidas destinadas a corregir la brecha de género en materia de $\mathrm{I}+\mathrm{D}+\mathrm{i}$.

Feminismo/s 34, diciembre 2019, pp. 289-315 
Del programa sectorial de estudios de las mujeres y del género a la integración de la perspectiva de género en los planes estatales de investigación científica y técnica y de innovación: avances, déficits y retos

Asimismo, también en el marco del Programa Estatal de I+D+i Orientada a los Retos de la Sociedad, además de lo contemplado específicamente en cada uno de los retos, se contempla la investigación en materia de género como un ámbito específico así como una variable transversal a incorporar en el desarrollo de las investigaciones orientadas a la búsqueda de soluciones en los distintos retos de la sociedad (Ministerio de Industria, Economía y Competitividad 2017, 56, 59, 72).

Por tanto, parece que, de inicio, hay una mayor inclusión de la igualdad de género y de la perspectiva de género en la formulación del Plan Estatal de I+D+i 2017-2020. Sin embargo, en la relación de los indicadores de gestión y seguimiento de las actuaciones del Plan Estatal, así como en las tablas de indicadores de cumplimiento de los objetivos y resultados asociados a los distintos Programas Estatales, no se contiene ninguno que de manera específica haga referencia a la evaluación y consecución de los objetivos relacionados con la implementación de la igualdad de género y la integración del análisis de sexo/ género, ni siquiera en la relación de los indicadores de objetivos y resultados del Programa Estatal de I+D+i Orientada a los Retos de la Sociedad (Ministerio de Industria, Economía y Competitividad 2017, 79-83). Igualmente, en los Programas de Actuación Anual de 2018 y 2019 del Plan Estatal de I+D+i 2017-2020 no se contiene ninguna mención relacionada con la inclusión de la igualdad de género y la integración de la perspectiva de género (Ministerio de Ciencia, Innovación y Universidades 2018, 2019). Por todo, no parece fácil que al finalizar su vigencia se hayan cumplido los objetivos previstos en la Hoja de Ruta del EEI para España en 2020.

\section{CONCLUSIONES: LA NECESARIA ADOPCIÓN DE DISPOSICIONES NORMATIVAS INTEGRALES MÁS EFICACES DE CONFORMIDAD CON EL EEI Y EL PRÓXIMO PROGRAMA MARCO «HORIZONTE EUROPA»: 2021-2027}

Como refleja este estudio, la consecución de la igualdad real y efectiva de mujeres y hombres en el marco español de investigación a lo largo de estas cuatro décadas ha sido muy limitada, de ahí que se mantenga un conjunto de desigualdades de género persistentes, especialmente la segregación de género en investigación y ciencia, el desequilibrio de género en cargos superiores

Feminismo/s 34, diciembre 2019, pp. 289-315 
Del programa sectorial de estudios de las mujeres y del género a la integración de la perspectiva de género en los planes estatales de investigación científica y técnica y de innovación: avances, déficits y retos

en la academia, los sesgos de género en el acceso a la financiación de la investigación, la investigación ciega al género y sesgada en el género, y la cultura organizativa y el proceso institucional ciegos al género y con sesgo de género, como reflejan los datos publicados en Científicas en Cifras 2017. Así, en la carrera investigadora se mantiene la infrarrepresentación de las mujeres en la categoría de mayor rango, con solo un $21 \%$ de mujeres entre el profesorado catedrático universitario, reflejándose una clara segregación vertical de género. Asimismo, la segregación vertical se manifiesta en los órganos de gobierno unipersonales, alcanzándose la mayor brecha de género a nivel de los Rectorados de las Universidades públicas, en los que las mujeres representan únicamente el $8 \%$. Por lo que se refiere a las comisiones técnicas de evaluación de convocatorias de ayudas a recursos humanos y a proyectos de I+D+i del Plan Estatal, solo el 53\% de las comisiones técnicas de recursos humanos y el $44 \%$ de las de proyectos tienen una presencia equilibrada de género. Y también se mantiene la brecha de género en las tasas de éxito de las ayudas a proyectos de $\mathrm{I}+\mathrm{D}+\mathrm{i}$ del Plan estatal, con 7 puntos porcentuales más para los IP varones (49\%) que para las IP mujeres (42\%) (Unidad de Mujeres y Ciencia 2018, 17-21).

Sin duda, la falta de la incorporación de la igualdad de género y de la integración de la perspectiva de género de manera real y efectiva en el marco español de investigación constituye un incumplimiento de lo establecido en la Ley 14/2011, de 1 de junio, de la Ciencia, la Tecnología y la Innovación y en la Estrategia Española de Ciencia y Tecnología y de Innovación 20132020, así como en el Programa Marco Horizonte 2020, en el Espacio Europeo de Investigación y en la Hoja de Ruta Española del Espacio Europeo de Investigación. Consciente de esta realidad y para impulsar y coadyuvar a su cumplimiento, el 23 de noviembre de 2018 se ha creado el Observatorio «Mujeres, Ciencia e Innovación» (OMCI), un órgano interministerial al que se le asignan, entre otras funciones: el seguimiento y análisis de las medidas y acciones adoptadas en materia de igualdad de género por los agentes del SECTI; la evaluación de las medidas adoptadas así como de la situación de las mujeres científicas, tecnólogas e investigadoras en España; y la formulación de recomendaciones y propuestas tendentes a mejorar los indicadores y sistemas de información relacionados con la igualdad de género en los ámbitos científicos y de investigación, la situación de las mujeres en

Feminismo/s 34, diciembre 2019, pp. 289-315 
Del programa sectorial de estudios de las mujeres y del género a la integración de la perspectiva de género en los planes estatales de investigación científica y técnica y de innovación: avances, déficits y retos

el SECTI, la adopción de medidas y actuaciones para avanzar en la consecución de la igualdad de género, incluidas las medidas de acción positiva, así como la integración del análisis de sexo/género en el contenido de las políticas, programas y proyectos de I+D+i (Observatorio «Mujeres, Ciencia e Innovación» 2019).

Y recientemente, el Real Decreto-ley 3/2019, de 8 de febrero, de medidas urgentes en el ámbito de la Ciencia, la Tecnología, la Innovación y la Universidad, ha introducido un nuevo párrafo en el apartado 4 de la Disposición adicional decimotercera de la LCTI para garantizar que los períodos de inactividad que afectan especialmente a las mujeres investigadoras por razones de conciliación de la vida personal y familiar no perjudiquen sus trayectorias, estableciéndose expresamente que los procedimientos de selección y evaluación del personal docente e investigador al servicio de las Universidades públicas y del personal investigador y de investigación al servicio de los OPIs de la Administración General del Estado, tendrán en cuenta las situaciones de incapacidad temporal, riesgo durante el embarazo, maternidad, guarda con fines de adopción, acogimiento, riesgo durante la lactancia y paternidad, de forma que las personas que se encuentren o se hayan encontrado en dichas situaciones tengan garantizadas las mismas oportunidades que el resto del personal que participa en los procesos de selección y evaluación, y su expediente, méritos y currículum vítae no resulten penalizados por el tiempo transcurrido en dichas situaciones (Disposición final primera).

Con todo, estas últimas medidas adoptadas de forma dispersa son manifiestamente insuficientes para garantizar que se cumplan efectivamente los objetivos previstos en materia de igualdad de género y de integración de la perspectiva de género en la Prioridad 4 de la Hoja de Ruta del EEI para España en 2020. Para su efectiva consecución será necesaria la regulación de forma sistemática mediante disposiciones normativas integrales más eficaces en la Ley de la Ciencia, la Tecnología y la Innovación y en la nueva Ley de Universidades de medidas efectivas que aborden los desequilibrios de género en las instituciones de investigación y los órganos de toma de decisiones e integren mejor la dimensión de género en las políticas, programas y proyectos de investigación, revisándose y adaptándose los procedimientos y criterios utilizados en las convocatorias públicas para evitar el sesgo de género y lograr un mayor equilibrio de género, mejorándose la integración de la

Feminismo/s 34, diciembre 2019, pp. 289-315 
Del programa sectorial de estudios de las mujeres y del género a la integración de la perspectiva de género en los planes estatales de investigación científica y técnica y de innovación: avances, déficits y retos

dimensión de género en las propuestas de financiación, incluyéndose indicadores específicos de evaluación y consecución de los objetivos relacionados con la implementación de la igualdad de género y la integración del análisis de sexo/género, y mejorándose los sistemas de monitorización, medición y rendición de cuentas, de conformidad con el EEI y tal como ya se plantea por la Comisión Europea en la propuesta del próximo Programa Marco de Investigación e Innovación «Horizonte Europa» (2021-2027) (European Commission 2018, 28).

\section{REFERENCIAS BIBLIOGRÁFICAS}

Comisión Interministerial de Ciencia y Tecnología (CICYT). Memoria de Actividades de I+D+I 2007. Madrid: 2009.

Comisión Interministerial de Ciencia y Tecnología (CICYT). Memoria de Actividades de I+D+I 2006. Madrid: 2008.

Comisión Interministerial de Ciencia y Tecnología (CICYT). Plan Nacional de Investigación Científica, Desarrollo e Innovación Tecnológica 2008-2011. Madrid: FECYT, 2007.

Comisión Interministerial de Ciencia y Tecnología (CICYT). Estrategia Nacional de Ciencia y Tecnología (ENCYT). Madrid: FECYT, 2007.

Comisión Interministerial de Ciencia y Tecnología (CICYT). Memoria de Actividades de $I+D+I$ 2005. Aprobada por el Consejo de Ministros el 14 de septiembre de 2007. Madrid: 2007.

Comisión Interministerial de Ciencia y Tecnología (CICYT). Memoria de Actividades de I+D+I 2004. Aprobada por el Consejo de Ministros el 21 de julio de 2006. Madrid: 2006.

Comisión Interministerial de Ciencia y Tecnología (CICYT). Memoria de Actividades de I+D+I 2003. Aprobada por el Consejo de Ministros el 29 de abril de 2005. Madrid: 2005.

Comisión Interministerial de Ciencia y Tecnología (CICYT). Memoria de Actividades de $I+D+I$ 2002. Aprobada por el Consejo de Ministros el 20 de febrero de 2004. Madrid: 2004.

Comisión Interministerial de Ciencia y Tecnología (CICYT). Memoria de Actividades de $I+D+I$ 2001. Aprobada por acuerdo de Consejo de Ministros de 2 de mayo de 2003. Madrid: 2003.

Feminismo/s 34, diciembre 2019, pp. 289-315 
Del programa sectorial de estudios de las mujeres y del género a la integración de la perspectiva de género en los planes estatales de investigación científica y técnica y de innovación: avances, déficits y retos

Comisión Interministerial de Ciencia y Tecnología (CICYT). Plan Nacional de Investigación Científica, Desarrollo e Innovación Tecnológica 2004-2007. Resumen. Aprobado por el Consejo de Ministros el 7 de noviembre de 2003. Madrid: 2003.

Comisión Interministerial de Ciencia y Tecnología (CICYT). Memoria de Actividades de I+D+I año 2000. Madrid: 2001.

Comisión Interministerial de Ciencia y Tecnología (CICYT). Memoria de Actividades de I+D en 1999. Aprobada por el Consejo de Ministros en su reunión de 30 de noviembre de 2001. Madrid: 2001.

Comisión Interministerial de Ciencia y Tecnología (CICYT). Memoria de Actividades de I+D en 1998. Aprobada por el Consejo de Ministros en su reunión de 30 de noviembre de 2001. Madrid: 2001.

Comisión Interministerial de Ciencia y Tecnología (CICYT). Plan Nacional de Investigación Científica, Desarrollo e Innovación Tecnológica 2000-2003. Resumen. Madrid: 2000.

Comisión Interministerial de Ciencia y Tecnología (CICYT). Memoria de Actividades del Plan Nacional de I+D en 1997. Aprobada por el Consejo de Ministros en su reunión de 4 de junio de 1999. Madrid: 1999.

Comisión Interministerial de Ciencia y Tecnología (CICYT). Memoria de Actividades del Plan Nacional de I+D en 1996. Aprobada por el Consejo de Ministros en su reunión de 28 de mayo de 1998. Madrid: 1998.

Comisión Interministerial de Ciencia y Tecnología (CICYT). Plan Nacional de I+D 1996-1999. Madrid: 1996.

Council of the European Union. Council Conclusions on the European Research Area Roadmap 2015-2020. Brussels: 19 May 2015.

Council of the European Union. Conclusion on progress in the European Research Area. Brussels: Competitiveness Council Meeting, 21 February 2014.

European Commission. Proposal for a regulation of the European Parliament and of the Council establishing Horizon Europe - The Framework Programme for Research and Innovation, laying down its rules for participation and dissemination. Brussels: 7.6.2018. COM (2018) 435 final.

European Commission. Implicit gender biases during evaluations: How to raise awareness and change attitudes? Workshop Report. Brussels: 30-31 May 2017. European Commission. The Spanish Roadmap for the European Research Area Development 2016-2020. Brussels: 2016. 
Del programa sectorial de estudios de las mujeres y del género a la integración de la perspectiva de género en los planes estatales de investigación científica y técnica y de innovación: avances, déficits y retos

European Commission. The EU Framework Programme for Research and Innovation. Horizon 2020. Excellent Science, Competitive Industries, Better Society. Vademecum on Gender Equality in Horizon 2020, RTD-B7 «Science with and for Society». Brussels: 26-02-2014, Rev. 21-03-2016.

European Commission. Science policies in the European Union. Promoting excellence through mainstreaming gender equality: A report from the ETAN expert working group on women and science. Brussels: Directorate-General for Research and Innovation, 2000.

Fundación Española para la Ciencia y la Tecnología (FECYT). Informe SISE 2006. Madrid: 2007.

GENDER-NET. Manuals with guidelines on the integration of sex and gender analysis into research contents, recommendations for curricula development and indicators. 2016.

League of European Research Universities (LERU). «Implicit bias in academia: A challenge to the meritocratic principle and to women's careers - And what to do about it». Advice Paper 23 (2018).

Ministerio de Ciencia, Innovación y Universidades. Programa de Actuación Anual Provisional 2019. Plan Estatal de Investigación Científica y Técnica y de Innovación 2017-2020. Ayudas para la financiación de la I+D+i en España. Madrid: 2019.

Ministerio de Ciencia, Innovación y Universidades. Observatorio «Mujeres, Ciencia e Innovación». Madrid: 2019.

Ministerio de Ciencia, Innovación y Universidades. Programa de Actuación Anual 2018. Plan Estatal de Investigación Científica y Técnica y de Innovación 20172020. Ayudas para la financiación de la I+D+i en España. Madrid: 2018.

Ministerio de Economía y Competitividad. Programa de Actuación Anual 2017. Plan Estatal de Investigación Científica y Técnica y de Innovación 2013-2016. Ayudas para la financiación de la I+D+i en España. Madrid: 2017.

Ministerio de Economía y Competitividad. Programa de Actuación Anual 2016. Plan Estatal de Investigación Científica y Técnica y de Innovación 2013-2016. Ayudas para la financiación de la I+D+i en España. Madrid: 2016.

Ministerio de Economía y Competitividad. Programa de Actuación Anual 2015. Plan Estatal de Investigación Científica y Técnica y de Innovación 2013-2016. Ayudas para la financiación de la I+D+i en España. Madrid: 2015. 
Del programa sectorial de estudios de las mujeres y del género a la integración de la perspectiva de género en los planes estatales de investigación científica y técnica y de innovación: avances, déficits y retos

Ministerio de Economía y Competitividad. Programa de Actuación Anual 2014. Plan Estatal de Investigación Científica y Técnica y de Innovación 2013-2016. Ayudas para la financiación de la I+D+i en España. Madrid: 2014.

Ministerio de Economía y Competitividad. Plan de Actuación Anual 2013. Plan Estatal de Investigación Científica y Técnica y de Innovación 2013-2016. Ayudas para la financiación de la I+D+i en España. Madrid: 2013.

Ministerio de Economía y Competitividad. Estrategia Española de Ciencia y Tecnología y de Innovación 2013-2020. Madrid: 2013.

Ministerio de Economía y Competitividad. Plan Estatal de Investigación Científica, Técnica y de Innovación 2013-2016. Madrid: 2013.

Ministerio de Educación y Ciencia. El Plan Nacional de Investigación Científica, Desarrollo e Innovación Tecnológica 2000-2003 en cifras. Madrid: Secretaría General de Política Científica y Tecnológica, 2005.

Ministerio de Hacienda y Administraciones Públicas. Acuerdo de Asociación de España 2014-2020. Madrid: 2014.

Ministerio de Industria, Economía y Competitividad. Plan Estatal de Investigación Científica y Técnica y de Innovación 2017-2020. Madrid: 2017.

Observatorio Español de I+D+i (ICONO) (Sistema Integral de Seguimiento y Evaluación (SISE). Informe SISE 2010. Análisis de las Convocatorias del Plan Nacional 2008-2011 correspondientes al año 2010. Madrid: FECYT, 2011.

Observatorio Español de I+D+i (ICONO) (Sistema Integral de Seguimiento y Evaluación (SISE). Informe SISE 2009. Análisis de las Convocatorias del Plan Nacional 2008-2011 correspondientes al año 2009. Madrid: FECYT, 2011.

Observatorio Permanente del Sistema Español de Ciencia-Tecnología-Sociedad (Sistema Integral de Seguimiento y Evaluación (SISE). Informe SISE 2008. Madrid: FECYT, 2009.

Unidad de Mujeres y Ciencia. Científicas en cifras 2017. Estadísticas e indicadores de la (des)igualdad de género en la formación y profesión científica. Madrid: Ministerio de Ciencia, Innovación y Universidades, 2018.

Unidad de Mujeres y Ciencia. Nota informativa sobre evaluación de la Integración del Análisis de Género en la Investigación (IAGI). Madrid: 2017.

Unidad de Mujeres y Ciencia. Científicas en cifras 2015. Estadísticas e indicadores de la (des)igualdad de género en la formación y profesión científica. Madrid: Ministerio de Economía, Industria y Competitividad, 2016. 
Del programa sectorial de estudios de las mujeres y del género a la integración de la perspectiva de género en los planes estatales de investigación científica y técnica y de innovación: avances, déficits y retos

Unidad de Mujeres y Ciencia. Científicas en cifras 2013. Estadísticas e indicadores de la (des)igualdad de género en la formación y profesión científica. Madrid: Ministerio de Economía y Competitividad, 2014.

Unión Europea. Decisión del Consejo, de 3 de diciembre de 2013, por el que se establece el Programa Específico por el que se ejecuta Horizonte 2020 - Programa Marco de Investigación e Innovación (2014-2020) y se derogan las Decisiones 2006/971/CE, 2006/972/CE, 2006/973/CE, 2006/974/CE y 2006/975/CE (2013/743/UE). DO L 347/965 de 20.12.2013.

Feminismo/s 34, diciembre 2019, pp. 289-315 